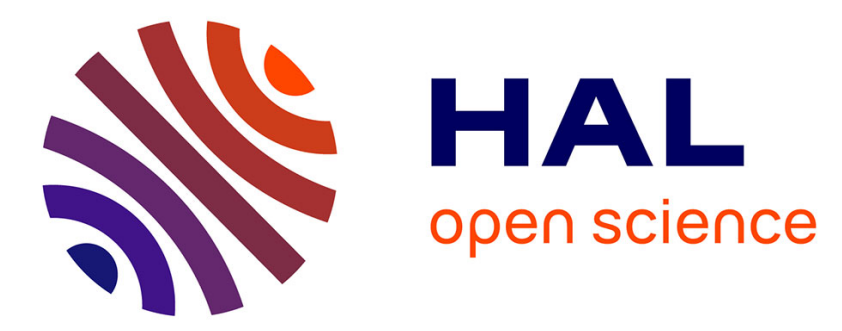

\title{
Statistical properties of focal conic textures in smectic liquid crystals
}

\author{
R. Bidaux, N. Boccara, G. Sarma, L. de Seze, P.-G. de Gennes, O. Parodi
}

\section{To cite this version:}

R. Bidaux, N. Boccara, G. Sarma, L. de Seze, P.-G. de Gennes, et al.. Statistical properties of focal conic textures in smectic liquid crystals. Journal de Physique, 1973, 34 (7), pp.661-672. 10.1051/jphys:01973003407066100 . jpa-00207427

\section{HAL Id: jpa-00207427 https://hal.science/jpa-00207427}

Submitted on 1 Jan 1973

HAL is a multi-disciplinary open access archive for the deposit and dissemination of scientific research documents, whether they are published or not. The documents may come from teaching and research institutions in France or abroad, or from public or private research centers.
L'archive ouverte pluridisciplinaire HAL, est destinée au dépôt et à la diffusion de documents scientifiques de niveau recherche, publiés ou non, émanant des établissements d'enseignement et de recherche français ou étrangers, des laboratoires publics ou privés. 


\title{
STATISTICAL PROPERTIES OF FOCAL CONIC TEXTURES IN SMECTIC LIQUID CRYSTALS
}

\author{
R. BIDAUX, N. BOCCARA, G. SARMA, L. DE SEZE \\ SPSRM, Orme des Merisiers, CEN de Saclay, BP nº 2, 91190 Gif-sur-Yvette, France \\ P. G. de GENNES \\ Collège de France, 11, place Marcelin-Berthelot, 75231 Paris Cedex 05, France \\ O. PARODI \\ Laboratoire de Physique des Solides, Université de Paris-Sud, 91400 Orsay, France
}

(Reçu le 24 janvier 1973, révisé le 21 mars 1973)

\begin{abstract}
Résumé. - Dans un spécimen smectique, les couches doivent être équidistantes, mais elles peuvent être courbées. Lorsque les conditions aux limites ne sont pas compatibles avec des couches planes, on obtient une texture dite à coniques focales. Nous considérons ici le cas usuel où chaque volume élémentaire de la texture est un cône. Les interstices entre ces cônes sont remplis par d'autres cônes plus petits, etc. Nous discutons certains aspects de ce remplissage itératif, et montrons qu'il doit se poursuivre jusqu'à une échelle très petite. Une estimation de l'énergie associée à la structure itérée indique qu'une texture à coniques focales est plus économique qu'un joint de grains, en accord avec les observations optiques usuelles. Nous discutons aussi la diffusion de la lumière par la structure itérée, au moyen d'un postulat de similarité. La dépendance angulaire de l'intensité diffusée à laquelle on arrive est singulière et son étude pourrait peut-être permettre de déterminer l'« indice de similarité $n$ » associé au processus itératif.
\end{abstract}

\begin{abstract}
In a smectic sample, the layers must be equidistant, but they may be curved. When the boundary conditions are not compatible with flat layers, one obtains a focal conic texture. We consider here the most familiar case where each unit in this texture fills a certain cone. The interstices between these cones are filled by smaller cones, etc. We discuss some features of this iterative filling of space, and show that it should persist down to a few molecular lengths. An estimate of the energy associated with the iterated structure indicates, that (in the absence of all external fields) focal conic textures are less expensive than grain boundaries, in agreement with the standard optical aspect of smectic samples. We discuss the scattering of light by an iterated focal conic texture, in terms of a simple scaling law; the resulting angular dependence of the scattered intensity is anomalous and may allow for a direct determination of the "scaling index " $n$ associated with the iterative process.
\end{abstract}

1. Introduction. - The present paper is concerned only with the simplest smectic phase, the so called smectic $\mathrm{A}$ in the classification of Sackmann and Demus [1]. In an ideal single domain of a smectic A, we have equidistant layers of width $a$. Each layer is a two dimensional liquid. The constituent molecules are on the average normal to the layers, and the system is optically uniaxial (Fig. 1a).

In practice, the arrangement of figure $1 a$ is obtained only if special precautions are used. In particular the boundary conditions at both sample surfaces must be controlled, and must be compatible with the layer stacking. A more complicated case is shown on figure $1 b$ where the conditions on both plates are different. An important practical question is to

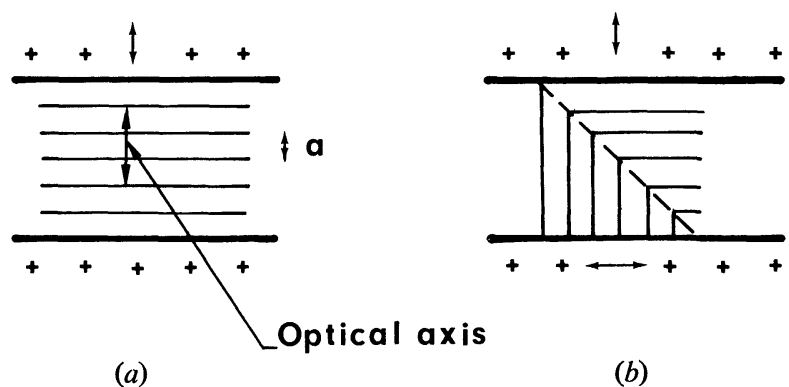

FIG. 1. - a) Texture of a smectic A between two parallel glass plates with identical boundary conditions. $b$ ) Case where the two plates impose different boundary conditions : the matching procedure represented here uses a grain boundary. In actual situations, this grain boundary will usually break up into focal conics, as explained in section (2.3). 


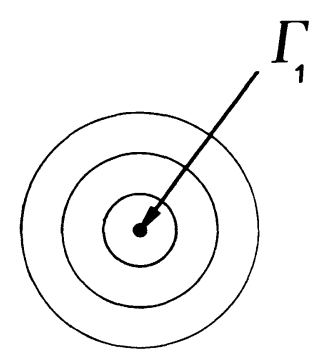

$\mathbf{Z}$
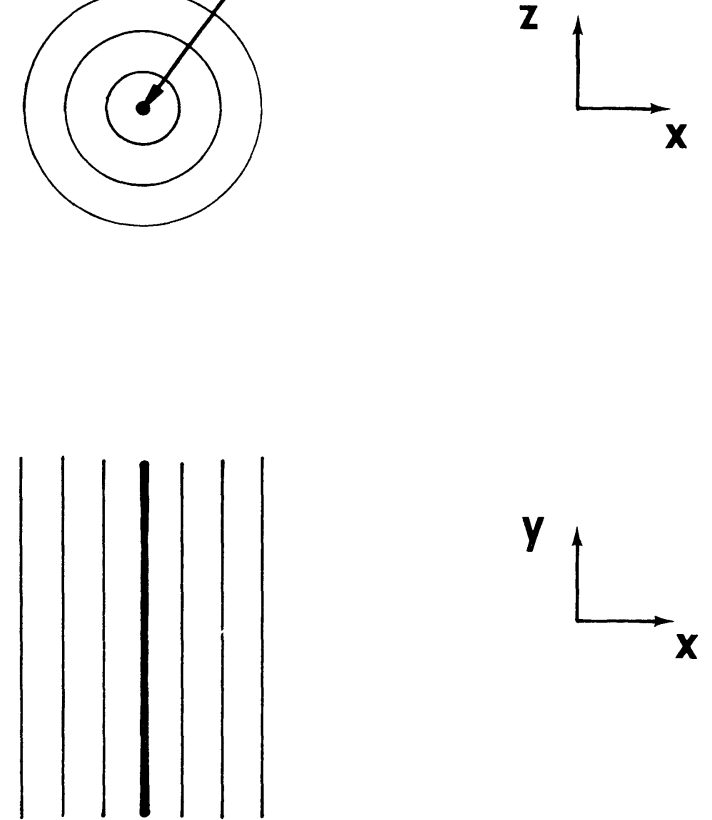

$\tau_{1}$

(a)

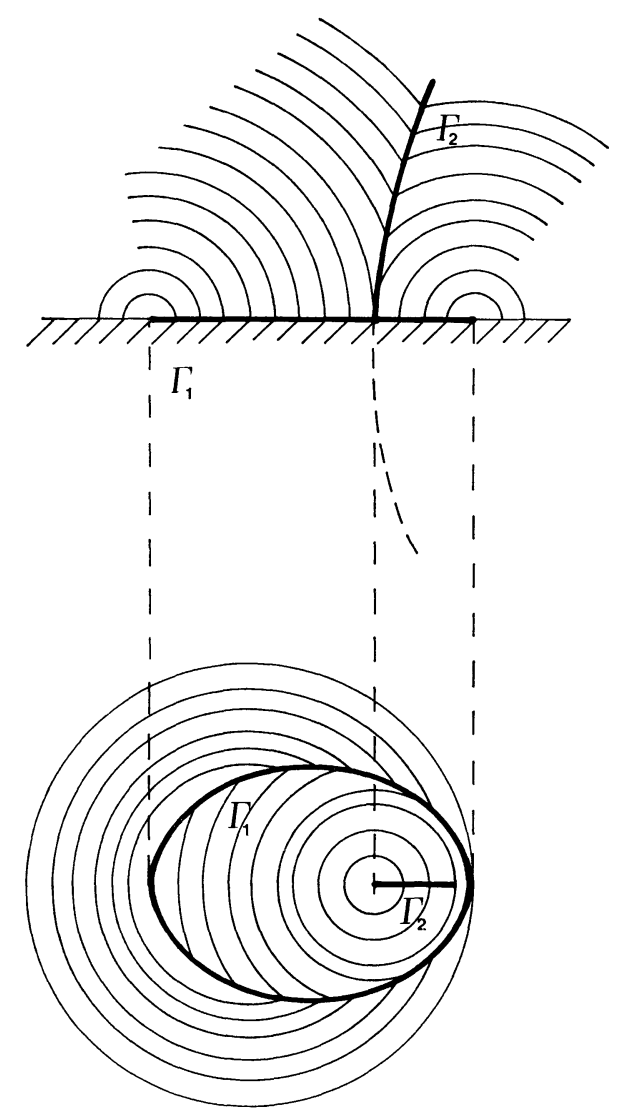

(c)

FIG. 2. - Focal conic textures. a) « Jelly roll» arrangement with one singular line $\Gamma_{1} . b$ ) Deformation of the jelly roll into a torus : two singular lines $\Gamma_{1}$ (circle) and $\Gamma_{2}$ (straight line) are involved. c) The general case : $\Gamma_{1}$ becomes an ellipse and $\Gamma_{2}$ is the conjugate hyperbola.

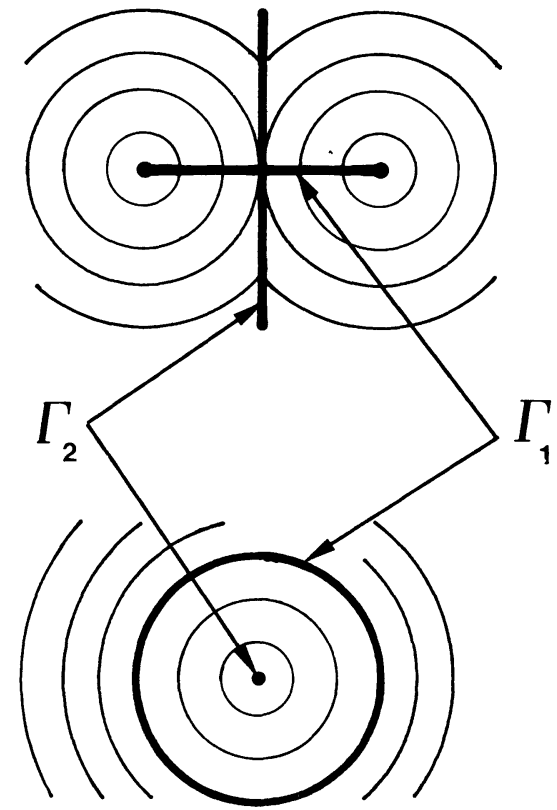

(b)

understand how a smectic system adjusts to conflicting requirements of this sort.

The solution shown on figure $1 b$ corresponds to a grain boundary, of thickness generally comparable to $a$, and of surface energy $\sigma\left({ }^{1}\right)$. However, grain boundaries are rarely observed in smectics : the adjustment to uneven surface conditions is usually obtained by a system of focal conics. This arrangement is described in the classic reviews by G. Friedel [2], by W. L. Bragg [3], and in a more recent series of papers by Y. Bouligand [4].

The essential features can be summarized as follows : the layers can be bent easily, and since they are liquid, they can slip on each other. This allows for a broad class of deformations, restricted only by the requirement of constant interlayer thickness. The simplest deformation is depicted in figure $2 a$ : the layers are folded into cylinders, with one singular line $\Gamma_{1}$ on the axis. But it is also possible to bend $\Gamma_{1}$ into a circle : each layer then becomes a torus, and we get the arrangement of figure $2 b$ with two singular lines : the circle $\Gamma_{1}$ and the axis $\Gamma_{2}$ of the torus. Finally the most general case is obtained by deforming $\Gamma_{1}$ into an ellipse, and $\Gamma_{2}$ into a conjugate hyperbola (Fig. 2c) : these two lines are called focal conics. The core structure and the energy of the focal conics have been discussed recently for one simple limiting case [5].

The smectic layers are set in agreement with the

(1) A simple calculation of the thickness, and of $\sigma$, can be carried out for the special case of a low angle grain boundary : this is explained in appendix A. 
above rules inside a cone of revolution, as shown on figure $3 a$. The apex of the cone is located on the hyperbola, and the base is the ellipse. If we want to fill a volume of definite shape, such as the square based pyramid of figure $3 b$, we begin by an ellipse of maximal size in the basal plane; then we fill the remaining interstices with smaller cones as shown on the figure.

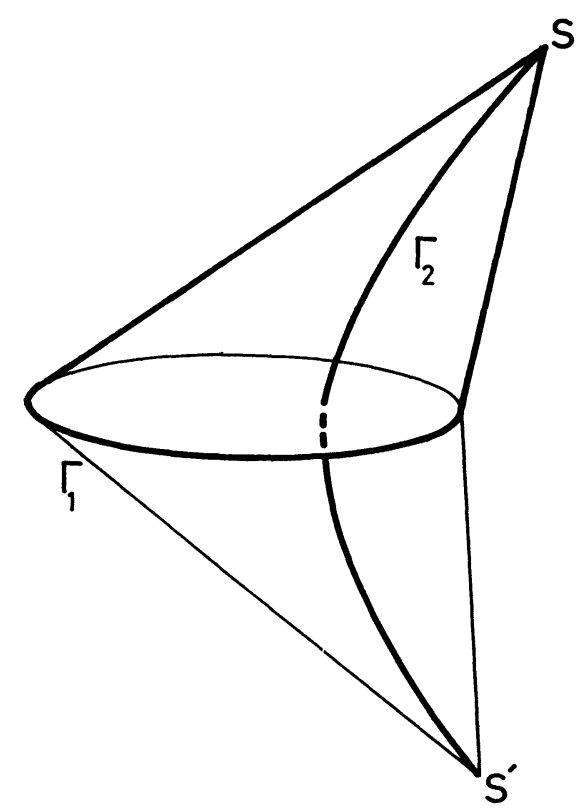

(a)

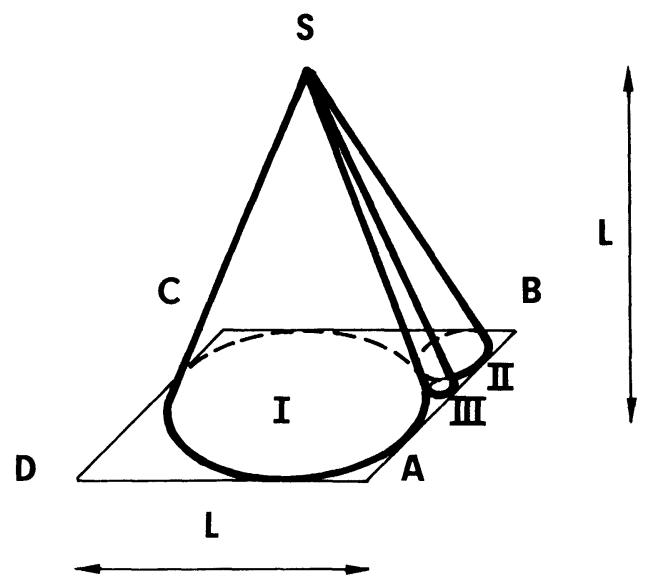

(b)

FIG. 3. - a) Example of a region of space filled by one focal conic arrangement : this is a cone of revolution based on the ellipse and with the apex $\mathbf{S}$ on the hyperbola. Very often, symmetrical cone (of apex $\mathrm{S}^{\prime}$ ) is also filled. b) Filling a pyramid $\mathrm{S}[\mathrm{ABCD}]$ of given shape by cones of the type shown in $(a)$. All cones have the same apex $S$ and are mutually tangent. On the figure are shown the first generation cone (I), and some examples of the second (II) and third generation (III). The iteration process goes on up to a very high number of generations.

All the cones are tangent along their common lines : this ensures that the orientation of the local optical axis is not discontinuous when going from one cone to the next.

The aim of this paper is to discuss some features of this «iterative filling of space» by tangent cones of decreasing size. We begin in section 2 by a geometrical discussion of the number of cones, of the perimeter of the associated focal conics, and of the remaining interstices, when the iteration is carried down to conics of some small size $\rho$. We find, by an estimation of the energies involved, that the physical limit $\rho^{*}$ should be very small $\left(-e g \rho^{*} \sim 10 a\right.$ ). Finally, we compare the energy of the iterated solution to that of a grain boundary, and show that the latter is higher.

Optical studies are in principle very adequate to probe the iterated structure : the optical wavelengths are much larger than $\rho^{*}$, but they are much smaller than the size $L$ of the largest conics (in most usual situations $L$ is comparable to the sample dimensions, and is thus of order $20 \mu$ or more). The spatial correlation functions which control the light scattering are then expected to have some rather simple scaling properties. We describe a conjectural form for these functions (or rather for their Fourier transforms) in section 3. Some further conjectures, analogies, and words of caution are listed in section 4 .

\section{Iterative filling of space with smectic material.} - 2.1 The APOLlONIAN PACKING OF CIRCLES. - Let us return to figure $3 b$, and consider for simplicity the limiting case where $S$ is far away from the basal plane $\mathrm{ABCD}$, so that the axis of each cone is nearly normal to the basal plane : then the ellipses are nearly circular. In the basal plane, we have a pattern of tangent circles shown on figure 4. Three mutually tangent circles define an interstice ; inside the interstice we can insert a fourth circle, tangent to the first three, thus defining three new interstices, etc.

An apparently similar iteration has already been used by one of us [6] in connection with properties of smectics under magnetic fields. However, the physical situation considered in [6] was very different : the smectic layers associated with one circle were assumed to be cylinders of revolution; of axis normal to the basal plane. This differs widely from the present problem, where the smectic layers become essentially parallel to the basal plane at levels higher than the radius $\rho$ of the conic, as is clear in figure $2 c$. As we shall see later, the discussion of the energies associated with the two models are not related. To make things quite definite, we may start with a periodic system of equal, large circles, and discuss the iteration inside one interstice between them. This iteration is known as the Apollonian packing of circles, and has been discussed in the mathematical literature [7], [8] $\left(^{2}\right)$. The results concerning the iteration to be presented below, were derived independently by us - without any mathematical rigor - but they do agree with the precise analysis of [7], [8].

(2) These references have been kindly quoted to us by professor B. Mandelbrot. 


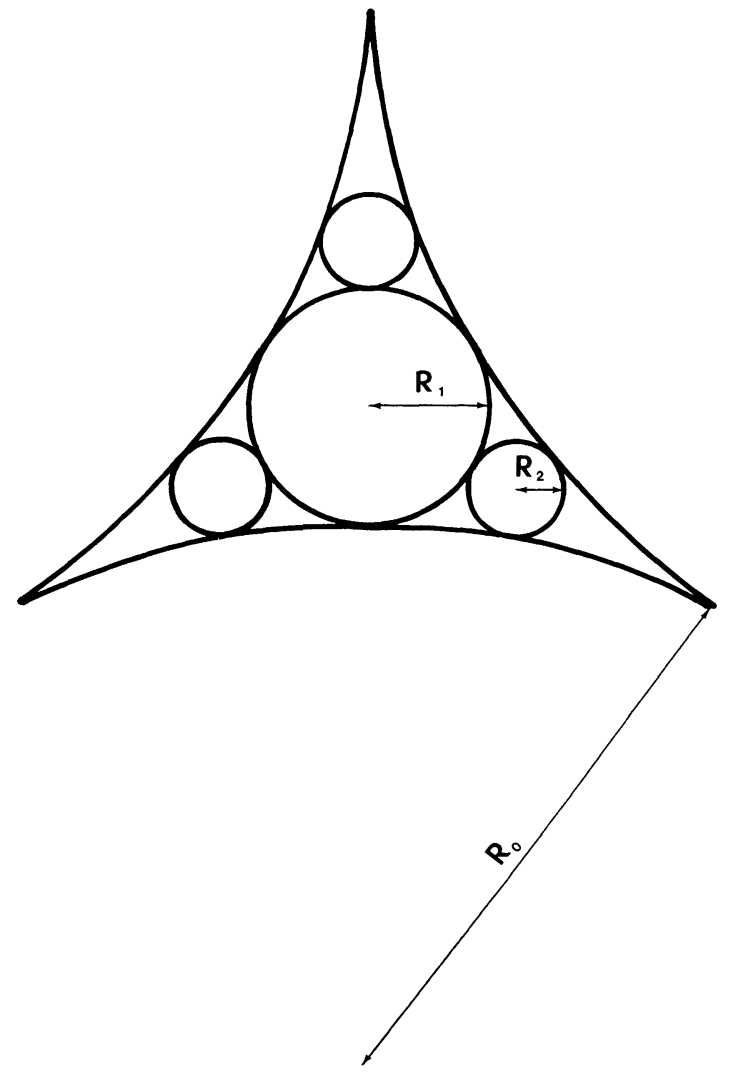

FIG. 4. - The iterative filling of figure $3 b$ as seen in the basal plane. For simplicity the apex $\mathbf{S}$ has been assumed to be far from the basal plane, so that all intersection are circles. Also, for the sake of illustration, a different set up has been chosen for the first generation, with three equivalent circles. (The choice of conditions at the first generation is not important for the scaling laws.)

The dimension of the largest circles, as well as the distance between the apex and the basal plane, will be associated with one macroscopic length $L$. Let us assume that we have carried out the iterative filling described above down to circles of radius $\geqslant \rho$ (where $\rho \ll L)$.

Let us call $g(\rho)$ the number of circles obtained at this stage. Clearly, $g$ depends only on the ratio $\rho / L$. As a function of $\rho, g(\rho)$ has the discontinuous aspect shown on figure 5. However, for $\rho / L \rightarrow 0, g$ may be approached by a continuous function. It is easy to see that

$$
\operatorname{Cte}\left(\frac{L}{\rho}\right)^{2}>g>\operatorname{Cte}\left(\frac{L}{\rho}\right)^{1 / 2} \text {. }
$$

The first inequality is obtained by observing that each circle counted in $g(\rho / L)$ has a radius $\geqslant \rho$, and an area $\geqslant \pi \rho^{2}$. All the circles are located in the initial interstice, of area $\alpha L^{2}$ where $\alpha$ is a numerical coefficient. Thus $g<\alpha / \pi(L / \rho)^{2}$. The second inequality is obtained by counting a subseries of circles represented on figure 6. It can be shown that the radius of the $m^{\prime}$ th circle in this subseries is of the form

$$
R_{m} \cong \frac{L}{\left(m+m_{0}\right)^{2}}
$$

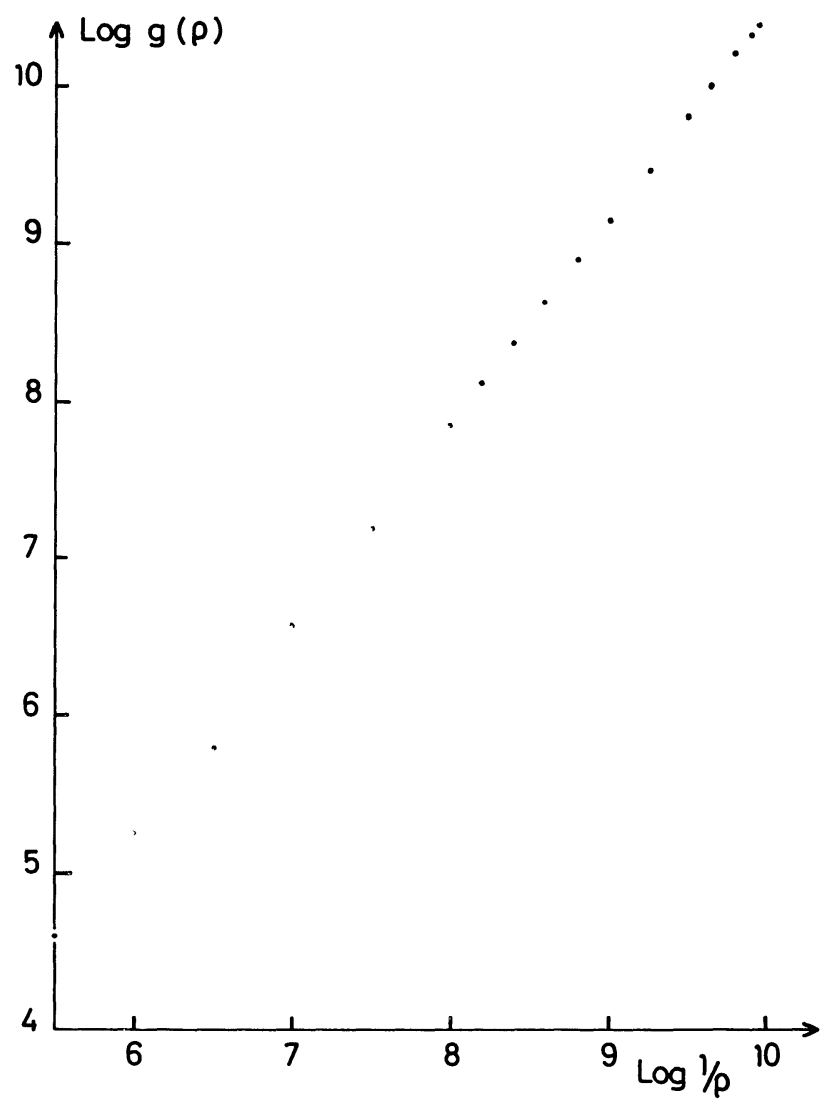

FIG. 5. - Number $g(\rho)$ of circles generated by the iteration process, and of radius larger than $\rho$. (The size of the circles of the first generation is called 1). Asymptotically for, $\rho \ll 1, g$ tends towards a power law.

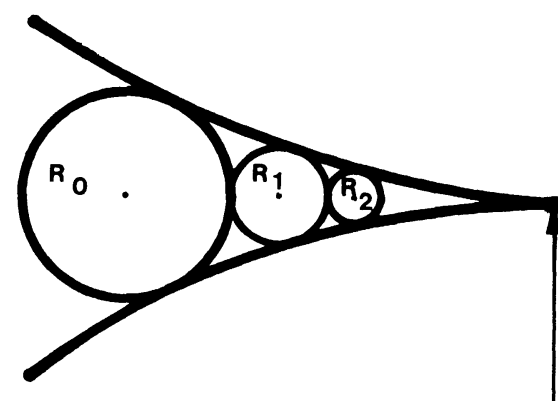

FIG. 6. - A partial iteration leading to a family of circles of radii $R_{0}, R_{1}, R_{2}, \ldots$ 
where $m_{0}$ is a numerical constant, depending on the choice of the first circle $\left(R_{1}\right)\left({ }^{3}\right)$. Stopping the series at $R_{m} \geqslant \rho$ and counting the number of terms, one can prove the second half of (2.1).

The inequalities (2.1) suggest that $g$ is of the form

$$
g=\operatorname{Cte}\left(\frac{L}{\rho}\right)^{n} \quad(L \gg \rho) .
$$

The exponent $n$ will be called the scaling index. We already know that $1 / 2 \leqslant n \leqslant 2$. Stronger inequalities are demonstrated in the references listed under [7b]. Numerical studies by Gilbert [8] gave $n \cong 1.3$. Similar calculations, carried out independently in [6], indicated $n \cong 1.32$. More detailed compilations are described in appendix $\mathrm{B}$, and give $n \sim 1.307$. In practice, to simplify the algebra, we shall often write $n=4 / 3$.

A number of useful auxiliary functions may be associated with $g$.

2.1.1 The total perimeter $P(\rho)$ of the circles generated by the iteration down to radius $\rho$. We have

$$
\begin{aligned}
& \mathrm{d} P=-2 \pi \rho \mathrm{d} g \\
& P(\rho)=\text { Cte } L^{n} \rho^{1-n} .
\end{aligned}
$$

2.1.2 The residual surface $\Sigma(\rho)$ is defined as the surface of the interstices which are left when the iteration is stopped at radius $\rho$. Whe have

$$
\begin{aligned}
& \mathrm{d} \Sigma=-\pi \rho^{2} \mathrm{~d} g \\
& \Sigma(\rho)=\operatorname{Cte} L^{n} \rho^{2-n} .
\end{aligned}
$$

Note that for $\rho \rightarrow 0$ the total perimeter $P(\rho)$ diverges (since $n>1$ ). On the other hand, the residual surface $\Sigma(\rho)$ goes to $\mathrm{O}$ when $\rho \rightarrow 0$. This was first proven by Kasner and Supnik [7a].

2.2 Physical Limit of THE ITERATION PROCESS. Let us now return to the three dimensional problem of figure $3 b$, and estimate the energies involved.

a) To each ellipse is associated an energy per unit length of the form $x K_{1}$, where $K_{1}$ is a Frank splay coefficient [9], and $x$ a number [5]. ( $x$ depends logarithmically on the radius of the conic, but we shall ignore this weak dependence). The total energy of one circle is thus $2 \pi \rho x K_{1}$.

b) To each hyperbola is also associated a line energy : for small $\rho$, the length of the hyperbola $(\sim L)$ is much larger than $\rho$. Thus at first sight we might think that the resulting energy is of order $K_{1} L$. But this is not correct : the only significant portions of the line are within a distance $\rho$ from the basal plane. Above this distance the smectic layers intersect each other at a very small angle on the line, and the energy drops rapidly [5]. The result is again of the form Cte $K_{1} \rho$. The sum of the contributions $(a)+(b)$ for

(3) The simple form (2.2) holds only when all circles $\left(R_{1}\right)$ $\left(R_{2}\right) \ldots$ are much smaller than $L$. a volume $L^{3}$ is thus of order

$$
F_{\text {line }}=\text { Cte } K_{1} P(\rho) \text {. }
$$

c) We must now consider the shape of the layers near the interstices which remain when we stop the iteration process. The total area of these interstices is $\Sigma(\rho)$. Also, the vertical size of the strongly distorted region above them is of order $\rho$. In all this region the dilatation of the smectic layers is of order unity, and the elastic energy per $\mathrm{cm}^{3}$ is comparable to the corresponding Young's modulus $\bar{B}$ [10]. Thus we get a contribution

$$
F_{\text {dist }}=\text { Cte } \bar{B} \rho \Sigma(\rho) \text {. }
$$

The line energy (2.6) tends to favor large $\rho$ values, while the distortion energy (3.7) favors small $\rho$ ' s. The optimal $\rho=\rho^{*}$, obtained by minimizing $F_{\text {line }}+F_{\text {dist }}$, and using eq. (2.4.5) is of the form

$$
\rho^{*}=\operatorname{Cte}\left(\frac{K_{1}}{\bar{B}}\right)^{1 / 2}
$$

$\left(K_{1} / \bar{B}\right)^{1 / 2}=\lambda$ is a molecular length $\left({ }^{4}\right)$ : we see that the iteration process should go down to a very small scale. The value of the free energy is

$$
\begin{aligned}
F_{\text {focal }} & \sim K_{1} P\left(\rho^{*}\right) \\
& \sim\left(\frac{L}{\rho^{*}}\right)^{n} K_{1} \rho^{*} .
\end{aligned}
$$

The discussion leading to eq. (2.7) is clearly oversimplified. In particular, the smaller conics will deform into polygons, for which the filling becomes perfect [4b]. But eq. (2.7) should remain qualitatively correct.

2. 3 COMPARISON BETWEEN GRAIN BOUNDARIES AND FOCAL CONICS. - A grain boundary is shown in figure 7 : at the boundary, the smectic planes from the

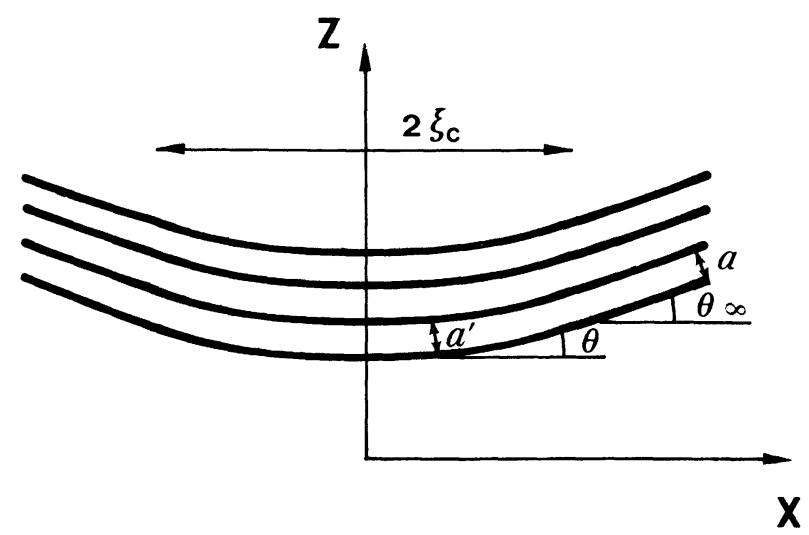

FIG. 7. - Structure of a symmetrical grain boundary. The thickness $2 \xi_{\mathrm{c}}$ is discussed in appendix $\mathrm{B}$, and is comparable to the interlayer distance $a$ (except for the case $\theta_{\infty} \rightarrow 0$ where $\left.\xi_{\mathrm{c}} \sim a / \theta_{\infty} \gg a\right)$.

(4) This argument fails near the smectic $A$-nematic transition température $T_{c}$ : when $T \rightarrow T_{c}, \bar{B} \rightarrow 0$ and $\lambda$ and $\rho^{*}$ become macroscopic lengths. The iteration process must then be stopped after a few generations. 
right half space make an angle $2 \theta_{\infty}$ with the planes from the left half space. The adjustment takes place in a microscopic length $\xi_{c}$, which is discussed in appendix B. The energy per unit area is $\sigma \sim\left(K_{1} B\right)^{1 / 2}$. Is it possible to solve the same adjustment problem with a suitable set of focal conics ? We describe in figure 8 a periodic pattern which is nearly adequate [11]. Many other patterns are also possible, and this

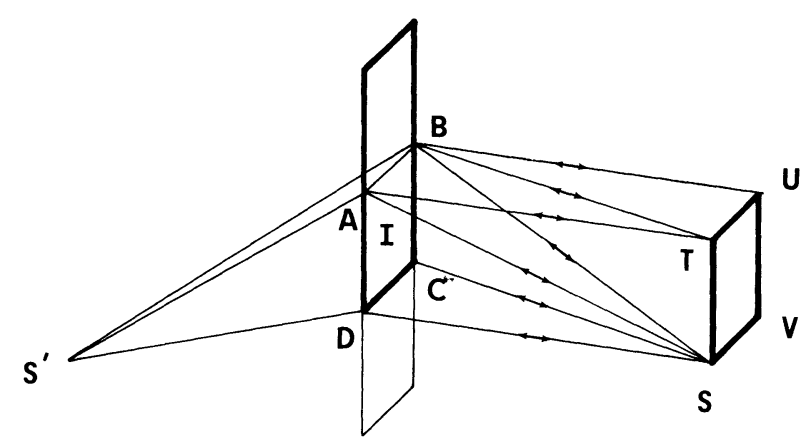

FIG. 8. - One possible substitute for the grain boundary of figure 7. The boundary plane is $\mathrm{ABCD}$. The pyramids $\mathrm{S}$ [ABCD] and $\mathrm{S}^{\prime}[\mathrm{ABCD}]$ are filled with focal groups as in figure $3 b$. The tetrahedron ABTS is essentially one single focal group, AB and TS being conjugated arcs. The same is true for the tetrahedron BCSV (not shown). The pyramid B[STUV] is filled with spherical layers centred on $B$. The directions of the local optical axis are shown by double arrows. The structure must be repeated periodically by translations parallel to $\mathrm{AB}$ and $\mathrm{AD}$.

particular one is chosen only for illustrative purposes. The arrangement is built as follows.

a) The square based pyramids such as S[ABCD] have their base in the nominal boundary plane. The median SI corresponds to the average optical axis in the right half space. Each of these pyramids is filled by iteration of cones as described above. On the left side we have a symmetrical pyramid $S^{\prime}[A B C D]$ : the smectic layers in both pyramids match smoothly in the basal plane. The distance between $S$ and the basal plane $L$ is comparable to the sample dimensions. The lateral dimension $\mathrm{AB}$ is expected to be a finite fraction of $L$.

b) Tetrahedra such as ABST can be described in a first approximation as two confocal arcs (AB and ST) inside which we have a single family of layers, with their normals touching both AB and ST $\left(^{5}\right.$ ).

c) Square based pyramids such as B[STUV] are filled with spherical layers centred on $B$.

With these rules the contacts between all adjacent polyhedra are smooth. The only remaining troubles are weak grain boundaries on surfaces such as STUV, which can be removed in turn by interating the whole procedure; since the angular discontinuity at this surface is much smaller (and equal to 0 on the average)

(5) We are indebted to M. Kleman for suggesting this possibility. this new iteration should converge rapidly as regards energies. In practice, the features at the STUV level may depend of the details of the sample boundaries, which are not far.

The energy associated with the model of figure 8 comes mainly from the first group of pyramids (the group (a) above) ; this is the only group where (in our approximate description) we find internal singular lines. The energy due to this group is estimated in eq. (2.9). The contribution of the other polyhedra $(b)$ and $(c)$, results from simple curvatures, of order $1 / L$, and is of order

$$
F_{\mathrm{bc}} \cong K_{1}\left(\frac{1}{L}\right)^{2} L^{3} \sim K_{1} L
$$

per polyhedron. This energy is proportional to $L$, while (2.9) is proportional to $L^{n}$ with $n>1$. Thus (2.9) is the dominant term, and we may write for the energy per unit area in the basal plane

$$
\tilde{\sigma} \cong \frac{F_{\text {focal }}}{L^{2}}=K_{1} L^{n-2}\left(\rho^{*}\right)^{1-n}
$$

We may now compare $\tilde{\sigma}$ and $\sigma$ :

$$
\begin{aligned}
\frac{\tilde{\sigma}}{\sigma} & \cong\left(\frac{K_{1}}{\bar{B}}\right)^{1 / 2} L^{n-2}\left(\rho^{*}\right)^{1-n} \\
& =\left(\frac{\rho^{*}}{L}\right)^{2-n} .
\end{aligned}
$$

Since the scaling index $n$ is smaller than 2, the exponent in (2.12) is positive $(2-n \sim 2 / 3)$ and the ratio $\tilde{\sigma} / \sigma$ is small. Typically if $\rho^{*}=100 \AA$ and $L=10 \mu$, $\tilde{\sigma} / \sigma \sim 1 / 100$. Thus, at least for the simple situation discussed here a grain boundary is metastable and tends to collapse into a set of focal conics $\left({ }^{6}\right)$. However, the concept of a grain boundary may remain convenient to guess the possible distortions of a smectic A under external constraints, provided that, at the end of the discussion, the grain boundary is replaced by a set of focal conics (i. e. Fig. 7 is replaced by Fig. 8). This idea is applied in appendix $\mathrm{C}$ (to discuss certain magnetic field effects). However, the discussion of appendix $\mathrm{C}$ is based on an assumption of complete equilibrium, which may be very hard to achieve. A more direct test of the iterative filling can be obtained, in principle, by optical scattering studies, to be described in the next section.

3. Light scattering by focal groups. - 3.1 DEFINITIONS. - Locally a smectic A is a uniaxial medium, the optical axis being normal to the layers. It is convenient to label this axis by a unit vector $\mathbf{n}(\mathbf{r})$,

(6) Eq. (2.12) also shows that it is favorable to have $L$ as large as possible : usually $L$ will be comparable to the sample size. Under fields, however, a natural unit of length may occur for $L$ (see appendix $C$ ). 
which we call the director. The dielectric tensor may be represented in dyadic form as

$$
\widehat{\varepsilon}(r)=\varepsilon_{\perp}+\varepsilon_{\mathrm{a}} \mathbf{n}: \mathbf{n}
$$

where $\varepsilon_{\perp}\left(\varepsilon_{\|}\right)$are the dielectric constants for a field $E$ normal (parallel) to $\mathbf{n}$, and $\varepsilon_{\mathrm{a}}=\varepsilon_{\|}-\varepsilon_{\perp}$. The intensity of light scattering from an incident beam of wave vector $\mathbf{k}_{0}$, polarization $\mathbf{c}$, scattered into $\left(\mathbf{k}_{1}, \mathbf{f}\right)$, is essentially proportional to

$$
\begin{aligned}
I_{\mathrm{if}}(\mathbf{q}) & =\int \varepsilon_{\mathrm{if}}\left(\mathbf{r}_{1}\right) \varepsilon_{\mathrm{fi}}\left(\mathbf{r}_{2}\right) \mathrm{e}^{\mathrm{i} \mathbf{q} \cdot \mathbf{R}_{12}} \mathrm{~d} \mathbf{r}_{1} \mathrm{~d} \mathbf{r}_{2} \\
(\mathbf{q} & \left.=\mathbf{k}_{0}-\mathbf{k}_{1}, \mathbf{R}_{12}=\mathbf{r}_{1}-\mathbf{r}_{2}\right) .
\end{aligned}
$$

The main assumption hidden behind eq. (3.1) is that the birefringence anisotropy (described here by $\varepsilon_{\mathrm{a}}$ ) must be small $\left(\varepsilon_{\mathrm{a}} \ll 1\right)$. This requirement is more stringent for studies on focal conics than for the more conventional studies on thermal scattering by single domains : $a$ ) in the larger focal domains we want to avoid any curvature of the light rays, $b$ ) multiple scattering must be ruled out. These demands may impose the choice of special materials (such as cholesterol esters) where $\varepsilon_{\mathrm{a}}$ is indeed small.

3.2 CONJECTURE ON THE FORM OF $I(q)$. - Let us now return to the system of cones shown on figure $3 b$. An exact calculation of the intensity $I(q)$ scattered by such a structure is clearly not feasible. We shall now describe a tentative argument leading to a simple form for $I(q)$ : near one circle of radius $\rho$ of the basal plane, we have a strongly perturbed region of volume $\Omega \sim \rho^{3}$ which can give rise to an intense scattering. Let us think of these regions as independent scatterers : this should be qualitatively correct, since correlations between the optical axes $\mathbf{n}$ at two points near two different interstices are weak. We may then write $I(\mathbf{q})$ in the form

$$
I(\mathbf{q}) \cong \int \Omega^{2}(\rho) F^{2}(\mathbf{q} \rho)[-\mathrm{d} g(\rho)]
$$

where $F(\mathbf{q} \rho)$ is the form factor of one scattering object normalized by $F(\mathrm{O})=1 .[-\mathrm{d} g]$ is always the number of objects of size between $\rho$ and $\rho+\mathrm{d} \rho$. The factor $\Omega^{2}$ expresses the fact that we have a double volume integral in (3.1). More physically, we may say that the scattering amplitude is linear in $\Omega$, and the intensity is thus quadratic in $\Omega$.

The detailed structure of the form factor $F$ [its dependence on the polarization directions $\mathbf{i}$ and $\mathbf{f}$ and on the direction of $\mathbf{q}$ ] is expected to be very complex. But, when all those angular parameters are fixed, $F$ is a function only of the dimensionless variable $q \rho$. Making use of eq. (2.3) for $g$, we get

$$
\begin{aligned}
I(q) & \cong L^{n} \int F^{2}(q \rho) \rho^{5-n} \mathrm{~d} \rho \\
& =\frac{L^{n}}{q^{6-n}} \int_{x_{\min }}^{x_{\max }} x^{5-n} F^{2}(x) \mathrm{d} x .
\end{aligned}
$$

The integration limits are

$$
\begin{aligned}
& x_{\min }=q \rho^{*} \ll 1 \\
& x_{\max }=q L .
\end{aligned}
$$

We can always replace $x_{\min }$ by zero. The upper bound $x_{\max }$ is large for the situations of main physical interest. (If $q L$ was of order unity, the scattering would be very sensitive to the details of the boundary conditions, sample shape, etc.). It is thus tempting to let $x_{\max }$ go to infinity. However, we must first make sure that the integration converges. This is a delicate point: the behaviour of $F(x)$ at large $x$ is controlled by the singularities inside each scattering object.

We shall assume here that the focal conic texture under consideration is perfect : the only singularities are the focal conics themselves. On the focal conics the scattering amplitude $\varepsilon_{\mathrm{if}(\mathrm{r})}$ has discontinuities of finite amplitude. As shown in appendix $\mathrm{D}$, this probably leads to a scattering intensity for one line behaving like $1 / q^{5}$. Then $F^{2}(x) \sim 1 / x^{5}$ for $x \gg 1$ and the integration $\int_{0}^{x_{\max }} \mathrm{d} x$ in eq. (3.3) may be extended to $x_{\max }=\infty$. We are thus led to :

$$
I(q)=\operatorname{Cte} L^{n} \frac{1}{q^{6-n}} .
$$

The main features contained in eq. (3.4) are : $a$ ) $I$ is proportionnal to $L^{n}$ (and not to $L^{3}$ as in usual systems). b) $I(q)$ is very strongly peaked at small angles $(q \rightarrow 0)$.

3.3 Discussion. - It may be of interest to compare (3.4) to the spontaneous thermal scattering (due to fluctuations in the density, the interlayer thickness, etc.) : as shown in [10], for general $\mathbf{q}$ values, this thermal contribution should be rather small - i. e. comparable to what it is in a liquid. We may put qualitatively, for a volume $L^{3}$

$$
I_{\mathrm{th}}(q) \sim L^{3} \frac{k_{\mathrm{B}} \mathrm{T}}{\bar{B}}
$$

where $\bar{B}$ is a Young's modulus. For smectic systems in usual conditions, we may say that $k_{\mathrm{B}} T / \bar{B} \sim a^{3}$, where $a$ is a molecular length. This then gives

$$
\frac{I}{I_{\mathrm{th}}} \sim \frac{1}{(q L)^{3-n}} \frac{1}{(q a)^{3}} .
$$

Taking $L=100 \mu, q^{-1}=1 \mu, a=20 \AA$, we obtain $I / I_{\text {th }} \sim 3000:$ the thermal effects appear to be negligible.

A more serious worry is related to the presence of defects in the texture. Our discussion above was based on the assumption that the layers inside each focal domain were ideally arranged : however, as shown recently by Bouligand [4], one does observe in practice local distortions which are incompatible with the rule of constant interlayer thickness. These distortions are probably realized through a system of dislocations [11]. 
The long range distortions present around certain dislocations in smectics have been analysed recently [12] : they could contribute significantly to the light scattering.

4. Concluding remarks. - Our description of focal conic textures is extremely primitive for various reasons :

a) Geometrical aspects : we have mainly focussed our attention on the filling of one pyramid by a large number of cones. Usually, such pyramids will not be the only building blocks of the texture : for instance, in the example of figure 8 , we needed two other building blocks : tetrahedra (which in a first approximation we treated as belonging to one single pair of focal conics) and pyramids with spherical layers. A much more detailed geometrical study would be necessary to define all typical building blocks and their internal structure.

b) Calculational aspects : the scaling laws of section 2 and the exact value of the exponent $n$ are probably independent of the various simplifications imposed on the model (such as replacing the ellipses by circles, etc.). But the conjectures which we have put forward, for magnetic field effects and for light scattering must be considered as very tentative.

c) Physical aspects : here, in our belief, the main trouble is related to the possible role of dislocations lines. When a finite density of these lines is present, the rule of constant interlayer thickness is relaxed, and the structures can adjust more flexibly. All our discussion of the minimal interstices, and of their size $\rho^{*}$, should probably be restated in terms of a dislocation field. However, we tend to believe that the estimate of the elastic energy (eq. (2.7)) will remain valid when this is done.

In spite of these complications, we are inclined to hope that the physical picture presented here is correct on the whole : we start with big objects, of size $L$ controlled by the sample dimensions or similar properties, and fill the interstices with smaller objects, iterating the process down to a very small length $\rho^{*}$. This iterative property is very reminiscent of the eddies occurring in a strongly turbulent flow [13]. The range $L \gg \rho \gg \rho^{*}$ is the range where simple scaling laws hold and is the analog of the "inertial subrange " in the turbulence problem.

Another interesting notion emerges when we compare the iterated focal conic texture in smectics and the domains occurring in ferromagnets, in ferroelectrics or in superconductors. Domain structures originate from the possible coexistence of two (or more) thermodynamic phases of equal free energy (e. g. spin up an spin down in a uniaxial ferromagnet). The characteristic thickness of the domains is usually well defined, and is of the form $(L a)^{1 / 2}$ where $a$ is a microscopic length : the distribution of sizes $\rho$ is not peaked towards small values of $\rho$.
This statement must be qualified, however, for normal and superconducting domains in a type I superconductor : to satisfy a certain stability requirement, the domains may have to "branch " down to small sizes, as suggested long ago by Landau [14]. However this branching is not observed in practice : the iteration is stopped at an early stage by physical cut-offs, as explained in [15]. For the smectics A, we have not been able to imagine similar cut-offs, and we believe that the iteration will proceed down to very small sizes $\rho^{*}$ : the optical experiments discussed in section 3 would be of great help to clarify this point.

Acknowledgments. - We have greatly benefited from conversations with Y. Bouligand, J. Friedel, M. Kleman and B. Mandelbrot.

Appendix A : structure of a low angle grain boundary. - We consider here a symmetrical grain boundary, where both sets of smectic planes intersect the boundary plane under the same angle $\left(\pi / 2-\theta_{\infty}\right)$ (Fig. 7) $\left({ }^{7}\right)$. We assume furthermore that the angle $\theta_{\infty}$ is small : in this limit, as we shall see, the structure of the grain boundary can be derived from continuum elasticity. Our discussion is a simple transposition of [5] (where focal conics are analysed) to a simpler one dimensional problem.

The free energy density is of the form

$$
F=\frac{1}{2} \bar{B} \gamma^{2}+\frac{1}{2} K_{1}\left(\frac{\mathrm{d} \theta}{\mathrm{d} x}\right)^{2}
$$

where $\gamma$ is the local dilatation of the interlayer distance. Assuming that there are no dislocations associated with the surroundings of the boundary, we must have $a / \cos \theta_{\infty}=a^{\prime} / \cos \theta$ where $a^{\prime}$ is the local interlayer distance. This gives

$$
\gamma=\frac{a-a^{\prime}}{a} \cong \frac{1}{2}\left(\theta_{\infty}^{2}-\theta^{2}\right) .
$$

It is then convenient to introduce the dimensionless variables $f=\theta / \theta_{\infty}$ and

$$
\tilde{F}=\frac{4 F}{\bar{B} \theta_{\infty}^{4}}=\frac{1}{2}\left(1-f^{2}\right)^{2}+\frac{1}{2} \xi_{\mathrm{c}}^{2}\left(\frac{\mathrm{d} f}{\mathrm{~d} X}\right)^{2}
$$

where $\xi_{\mathrm{c}}=2 \lambda / \theta_{\infty}$ and $\lambda=\left(K_{1} / \bar{B}\right)^{1 / 2}$. In the limit of interest $\xi_{\mathrm{c}}$ is much larger than $\lambda$, and this justifies the use of a continuum theory. The energy per unit area of the grain boundary is

$$
\sigma=\int_{-\infty}^{\infty} F \mathrm{~d} X
$$

Minimising $\sigma$ with respect to $f$, one is led to a non linear equation of the Landau Ginsburg type

$$
-2 f\left(1-f^{2}\right)-\xi_{c}^{2} \frac{d^{2} f}{d x^{2}}=0 .
$$

(7) A symmetrical grain boundaries must always contain some dislocations. 
With the first integral (correctly adjusted to the boundary conditions)

$$
\xi_{\mathrm{c}} \frac{\mathrm{d} f}{\mathrm{~d} x}=1-f^{2} .
$$

This gives

$$
f=\tanh \frac{x}{\xi_{\mathrm{c}}}
$$

The thickness of the boundary is thus of order $2 \xi_{c}$. The energy $\sigma$ is conveniently calculated from (A.4), (A.3) and (A.6), as

$$
\begin{aligned}
\sigma & =\frac{\bar{B}}{4} \theta_{\infty}^{4} \xi_{\mathrm{c}}^{2} \int_{-\infty}^{\infty}\left(\frac{\mathrm{d} f}{\mathrm{~d} x}\right)^{2} \mathrm{~d} x \\
& =\frac{\bar{B}}{4} \theta_{\infty}^{4} \xi_{\mathrm{c}} \int_{-1}^{1} \mathrm{~d} f\left(1-f^{2}\right) \\
& =\frac{2}{3} \bar{B} \lambda \theta_{\infty}^{3} .
\end{aligned}
$$

Eq. (A.8) applies only when $\theta_{\infty} \ll 1$. However, it also gives us a qualitative estimate of the energy for a large angle grain boundary, namely $\sigma=\mathrm{Cte} \bar{B} \lambda$ : This estimate is used in section 2 .

Appendix B : Calculation of the scaling index $n$. In this appendix, we describe first two simplified geometrical problems where an index $n$ can be defined and computed exactly. Then, we proceed to the actual problem, and devise an estimation of $n$ based on computer techniques.

The first simplified model is shown on figure 9 : instead of circles, we deal with equilateral triangles; after $m$ iterations we have 1 triangle in the first generation, 3 triangles in the second generation, etc., and $3^{m-1}$ triangles in the $m$ th and last generation. Thus,

$g=1+3+3^{2}+\cdots+3^{m-1}=\frac{1}{2}\left(3^{m}-1\right) \sim \frac{1}{2} 3^{m}$.

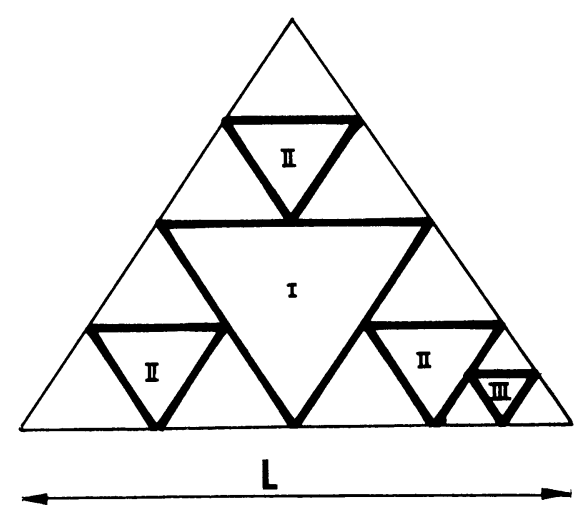

FIG. 9. - Simplified model for the iteration, based on equilateral triangles rather than on circles I, II, III represent successive generations.
The lateral dimension of the smallest triangles is

$$
\rho=\frac{L}{2^{m}}
$$

Thus

$$
m \ln 2=\ln \frac{L}{\rho}
$$

and

$$
g \sim \frac{3}{2}\left(\frac{L}{\rho}\right)^{m}
$$

with

$$
n=\frac{\ln 3}{\ln 2}=1.585
$$

A similar model is obtained by inscribing first a hexagon in the initial triangle at the first generation, leaving three equal equilateral triangles which are in turn filled with three hexagons at the second generation, etc. At each successive generation, the linear size of the hexagons is divided by a factor 3 , so that, for the $m$ th generation, the number of hexagons is $3^{m-1}$ while the lateral dimension of these hexagons is

$$
\rho=\frac{L}{3^{m}}
$$

It is then a simple matter to prove that

$$
g \sim \operatorname{Cte}\left(\frac{L}{\rho}\right)^{n}
$$

with

$$
n=\frac{\ln 3}{\ln 3}=1
$$

The triangular model underestimates the filling. The hexagonal model overestimates it. Thus we expect that the correct value of $n$ is intermediate between (B.4) and (B.6).

A more mathematical discussion of similar models has been carried out by A. Eggleton [7c].

Let us now return to the full problem of figure 4, where we choose to start with three equal circles of radius 1 , and fill the interstice between them by successive generation of circles.

Once more the number of circles belonging to the $m$ th generation will be $3^{m-1}$, but after the second generation, the radii of circles belonging to a given generation are no longer equal ; in fact, the $m$ th generation exhibits $\left[3^{m-2}+1\right] / 2$ different possible radii for its $3^{m-1}$ circles. Therefore, it is necessary to resort to computer calculations : our calculations are similar in spirit, but different in extension, with the earlier ones of [8] and [6].

For each "generation" of circles, all the possible radii have been calculated, together with the number of circles attached to each radius (in fact 3 or 6). In our calculation, all circles of radius $>\exp (-10)$ have 
been generated (some of them had to be looked for as far as the 50th generation).

Figure 5 shows a plot of $[\ln g(\rho)]$ vers. $\ln 1 / \rho$. The existence of a scaling index seems undisputable, at least in the region of values of $\rho$ considered. A linear adjustment corresponding to the interval $8<\ln$ $1 / \rho<10$ where a maximum density of data is available leads to the following result:

$$
\ln [g(\rho)]=n \ln \left(\frac{1}{\rho}\right)-k
$$

with $n \simeq 1.3069$ and $k \simeq 2.6000$.

\begin{tabular}{clll}
\hline $\ln 1 / \rho$ & \multicolumn{1}{c}{6} & \multicolumn{1}{c}{6.5} & \multicolumn{1}{c}{7} \\
- & - & - & - \\
$n(\rho)$ & 1.312 & 1.2863 & 1.3125 \\
$\ln 1 / \rho$ & 8.8 & 9 & 9.25 \\
$n(\rho)$ & 1.3083 & 1.3068 & 1.3066
\end{tabular}

One can see that the dispersion of $n(\rho)$ about the adjusted value $n=1.3069$ is very small, even for the values of $\ln 1 / \rho$ which have not been taken into account for the adjustment.

As pointed out above, the difficulty in proving the existence and establishing the exact value of the index $n$ lies in the fact that, for each generation of circles, there

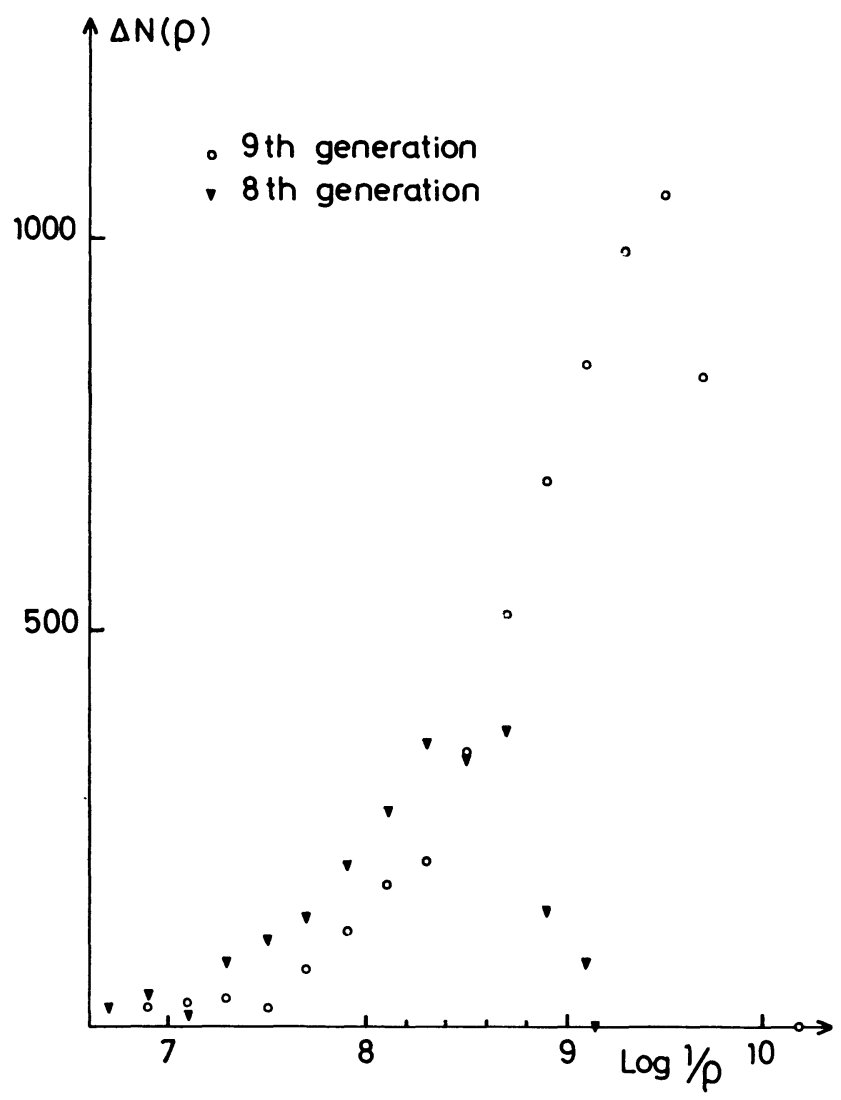

FIG. 10. - Distribution of sizes ( $\rho$ ) for the circles of the Apollonian problem belonging to one same generation. Note the broad spectrum of $\rho$ values. The largest $\rho$ 's are associated with the special iteration process of figure 6 .
This result can be put under the alternative form :

$$
g(\rho)=\left(\frac{\rho_{0}}{\rho}\right)^{n} \quad \text { with } \quad \rho_{0} \simeq 0.1368\left(^{8}\right)
$$

We may also define a «local» value of the index $n$ from the ratio

$$
n(\rho)=\frac{\ln g(\rho)+k}{\ln 1 / \rho}
$$

where $k=2.6000$. The results are shown in the following table :

\begin{tabular}{lllll}
\multicolumn{1}{c}{7.5} & \multicolumn{1}{c}{8} & \multicolumn{1}{c}{8.2} & \multicolumn{1}{c}{8.4} & \multicolumn{1}{c}{8.6} \\
- & - & - & - & - \\
1.3049 & 1.3074 & 1.3083 & 1.3070 & 1.3062 \\
9.5 & 9.7 & 9.8 & 9.9 & 9.95 \\
1.3076 & 1.3072 & 1.3075 & 1.3072 & 1.3067 \\
\hline
\end{tabular}

exists a wide distribution of the radii ; figure 10 shows this distribution, computed for the 8th and 9th generation : $\Delta N(\rho)$ represents the number of circles of a given generation having radii belonging to a given interval of width 0.2 in $\log 1 / \rho$ (the corresponding point is plotted at the center of the interval). A formulation of $\Delta N(\rho)$, even empirical, in terms of $\rho$ or $\log 1 / \rho$, would be most welcome.

Appendix C : Competition between wall alignment and field alignment in a smectic $A$. - We consider a semi infinite medium filled with smectic $\mathrm{A}$, under an applied magnetic field $\mathbf{H}$. The boundary conditions at the limiting wall impose an optical axis normal to $\mathbf{H}$. How does the smectic adjust to these conflicting requirements ? If we had a nematic fluid, the adjustment would take place gradually in a thickness

$$
\xi_{\mathrm{H}}=\left(\frac{K}{\chi_{\mathrm{a}}}\right)^{1 / 2} \frac{1}{H},
$$

where $K$ is a Frank constant and $\chi_{\mathrm{a}}$ is the diamagnetic anisotropy [16]. With a smectic $A$ the problem is much more complex, and the answer which we shall describe here must be considered as tentative.

The geometry is shown on figure 11. One possible method of adjustment (discussed in [6] for a similar problem) involves grain boundaries. Presumably the least expensive grain boundaries would be symmetrical, with the smectic planes at $\pm 45^{\circ}$ from the boundary plane as postulated on the figure. We would then have prismatic regions extending to a distance $L$ inside the smectic. Each of these regions is oriented unfavorably with respect to the field, and contributes an energy

(8) $\rho_{0}$ is not very different from the radius of the first inner circle. 


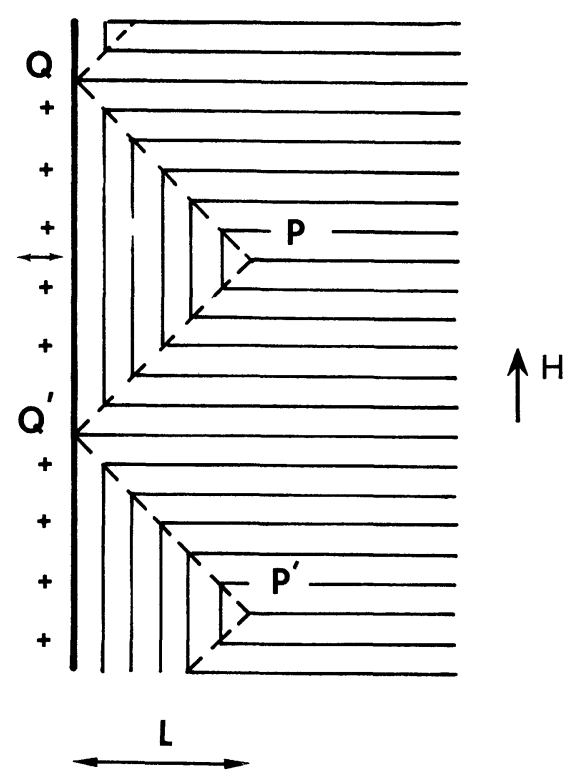

FIG. 11. - Competition between wall alignment and field alignment : the figure shows a naive model using grain boundaries $\mathrm{PQ}, \mathrm{QP}^{\prime}$, etc... A more plausible model is obtained by replacing each grain boundary by a system of focal conics, as in figure 8 .

$\chi_{\mathrm{a}} H^{2} / 2$ per $\mathrm{cm}^{3}$. The cross section area of one prism is $L^{2}$, and the length covered by it is $2 L$. Thus the magnetic energy per unit area of wall is

$$
F_{\text {mag }}=\frac{1}{2} \chi_{\mathrm{a}} H^{2} \frac{L^{2}}{2 L}=\frac{1}{4} \chi_{\mathrm{a}} H^{2} L \text {. }
$$

The surface energy of the grain boundaries is $\sigma / \mathrm{cm}^{2}$ of boundary, or (since the boundaries are at $45^{\circ}$ from the wall) it is, per $\mathrm{cm}^{2}$ of wall :

$$
F_{\mathrm{s}}=\sqrt{2} \sigma .
$$

Finally we have singular lines in $\mathrm{P}, \mathrm{P}^{\prime}$, etc. and in $\mathrm{Q}, \mathrm{Q}^{\prime}$, etc. Each of these lines will have an energy per unit length of order $K$ (where $K$ is always a Frank constant) and the system of lines will give a contribution per unit area of wall :

$$
F_{\mathrm{L}}=\frac{K}{L}
$$

Minimising the sum $F_{\text {mag }}+F_{\mathrm{S}}+F_{\mathrm{L}}$ we would obtain $L=2 \xi_{\mathrm{H}}$, i. e. the effects of the wall would be of comparable range in a nematic and in a smectic $\mathrm{A}$. One major difference occurs, however, if we compare the energies : the surface term $F_{\mathrm{S}}$ is dominant here, and is very large when compared to $F_{\text {mag }}$ or $F_{\mathrm{L}}$ : this suggests that arrangements of lower energy might exist.

At this stage we recall the discussion of section 2 : each grain boundary is probably unstable, and should break down in a system of focal conics, more or less similar to the model of figure 8 . We then make the following assumptions :

a) In spite of the complications introduced by the focal conics, the average alignment directions inside or outside the prisms are not far from the simpler picture of figure 11. This means in particular that (C.1) remains valid.

b) We ignore any special end effect occuring at the points $\mathrm{P}, \mathrm{P}^{\prime}, \ldots$ or $\mathrm{Q}, \mathrm{Q}^{\prime}, \ldots$ This means that we drop out the analog of $F_{\mathrm{L}}$ for a focal conic system. The validity of this assumption is extremely dubious. But any refinement on this point would require a considerable effort.

Accepting (a), (b), and (c) we are led to minimize an energy of the form

$$
F \cong \chi_{\mathrm{a}} H^{2} L+K_{1}\left(\rho^{*}\right)^{1-n} L^{n-2}
$$

In eq. (C.4) and in all what follows, all numerical coefficients are purposely ignored. The minimum of $F$ corresponds to

$$
\begin{aligned}
L^{n-3} & =\frac{\chi_{\mathrm{a}} H^{2}}{K_{1}}\left(\rho^{*}\right)^{n-1}=\xi_{\mathrm{H}}^{-2}\left(\rho^{*}\right)^{n-1} \\
L & =\xi_{\mathrm{H}}^{2 / 3-n}\left(\rho^{*}\right)^{1-n / 3-n} .
\end{aligned}
$$

Putting $n=4 / 3$ this would correspond to

$$
L=\xi_{H}^{6 / 5}\left(\rho^{*}\right)^{-1 / 5}
$$

The penetration depth $L$ is then proportional to $H^{-6 / 5}$. In view of the tentative prediction (C.6) it appears tempting to probe the behaviour of a smectic $\mathrm{A}$ under the conflicting actions of a wall and of a field. However, the arrangement would have to be prepared reversibly: even by forming the smectic phase from a nematic (or isotropic) phase by slow cooling under $H$, this reversibility may be prohibitively difficult to obtain : we may always observe growth figures rather than equilibrium figures.

For the opposite case (where the layers must be normal to the wall, and the field is also normal to the wall) other modes of adjustment are conceivable. One of them has been suggested by Y. Bouligand (private communication): in this mode the ellipses are all circular, and are in the plane of the surface. The hyperbolae are straight lines normal to the surface. The filled region associated with one pair of conics is a cylinder of revolution, based on the circle. These cylinders fill all the available space by Appolonian packing. (Note that no polygons are required by such a case.) Eq. (C.4) (C.6) remain valid, $L$ being now the size of the largest circles in the structure. This is also the thickness of the perturbed layer : thus, in spite of the geometrical differences, our estimate of this thickness is the same for both models.

Appendix D : Qualitative discussion of the scattering of light by one focal conic in the limit $q \rho \gg 1$. - We shall discuss here the scattering amplitude $A$ corresponding to eq. (3.1)

$$
A(\mathbf{q})=\int \varepsilon_{\mathrm{if}}(\mathbf{r}) \mathrm{e}^{\mathrm{i} \mathbf{q} \cdot \mathbf{r}} \mathrm{d} \mathbf{r}
$$


$\varepsilon_{\mathrm{if}}(\mathbf{r})$ is discontinuous on each focal conic line : this implies a certain form of decrease of $A(\mathbf{q})$ at large $q$, which we want to analyse. We consider one singular line, with a radius of curvature $\rho$ which is large compared with $1 / q$ : then the main contributions to $A(q)$ will come from a tube of radius $\sim 1 / q$ surrounding the line, and the interrelations of different lines are not expected to be very important.

Let us start by a straight line (along $\mathrm{z}$ ) and use semi polar coordinates $(\mathrm{r}, \varphi, \mathrm{z})$. The director $\mathbf{n}$ has one component $n_{\mathrm{z}}$ which is independent of the observation point $\left(n_{\mathrm{z}}=\cos \theta_{\infty}\right)$, and two variables components

$$
\begin{aligned}
& n_{\mathrm{x}}=\sin \theta_{\infty} \cos \varphi \\
& n_{\mathrm{y}}=\sin \theta_{\infty} \sin \varphi
\end{aligned}
$$

$\varepsilon_{\text {if }}$ is a quadratic function of the components of $\mathbf{n}$. It will contain terms proportional to $\cos \varphi, \sin \varphi$, $\cos 2 \varphi, \sin 2 \varphi$. The qualitative features of the results are the same for all these terms. For definiteness we shall discuss the $(2 \varphi)$ terms, and write them in the form

$$
\varepsilon_{\text {if }}=C \cdot \cos 2\left(\varphi-\varphi_{0}\right) .
$$

The integral (D.1) will vanish if $q_{\mathrm{z}} \neq 0$. Let us thus put $\mathbf{q}$ normal to the line and (for instance) along $\mathrm{x}$. We must compute the two dimensional integral

$X(q)=C \int_{0}^{2 \pi} \mathrm{d} \varphi \int_{0}^{\infty} \mathrm{rdr} \mathrm{e}^{\mathrm{i} q \mathrm{r} \cos \varphi} \cos 2\left(\varphi-\varphi_{0}\right) \mathrm{e}^{-\varepsilon r}$.

The last factor $\mathrm{e}^{-\varepsilon \mathrm{r}}$ (with a positive, small $\varepsilon$ ) is introduced to ensure convergence, and we shall take the limit $\varepsilon \rightarrow 0$ in the final results. Integrating first on $\mathrm{r}$, one obtains

$$
X=C \int_{0}^{2 \pi} \mathrm{d} \varphi \cos 2\left(\varphi-\varphi_{0}\right) \frac{1}{(\varepsilon-\mathrm{i} q \cos \varphi)^{2}}
$$

This integral can be evaluated by putting $z=e^{i \varphi}$. The result (for $\varepsilon \rightarrow 0$ ) is

$$
X=-\frac{4 \pi C}{q^{2}} \cos 2 \varphi_{0}
$$

Let us now turn to the physical case of a curved line (Fig. 12) : in the limit $q \rho \gg 1$ the only important regions will be those where the line is normal to q. Let $o$ be the point of the line where this condition is satisfied, oz the tangent to the line, xoz the osculator plane. The wavevector $q$ has two non vanishing component $q_{\mathrm{x}} q_{\mathrm{y}}$. To a good approximation we may write

$$
\varepsilon_{\mathrm{if}}(\mathrm{xyz})=\tilde{\varepsilon}_{\mathrm{if}}(u, \mathrm{y})
$$

where $\tilde{\varepsilon}$ is calculated as in (D.3) for an observation point at the distance $\left(u^{2}+y^{2}\right)^{1 / 2}$ from the line. The length $u$ is shown on figure 12 . For $\mathrm{x}, \mathrm{z} \ll \rho$ we may put

$$
u \cong \mathrm{x}-\frac{\mathrm{Z}^{2}}{2 \rho} \text {. }
$$

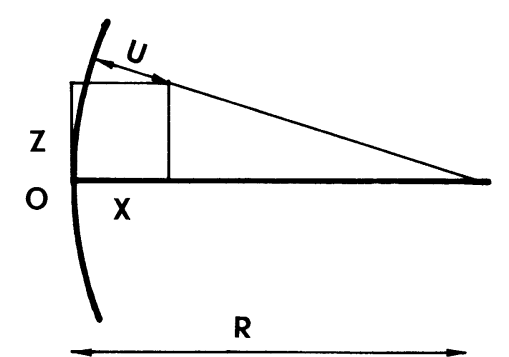

FIG. 12. - Geometrical basis for the calculation of the scattering by one curved focal conic.

The amplitude $A(\mathbf{q})$ may then be written as

$$
\begin{aligned}
A(\mathbf{q}) & =\int \mathrm{dz} \int \mathrm{d} u \mathrm{dy} \mathrm{e}^{\mathrm{i} q_{\mathrm{x}}\left(u+\mathrm{z}^{2} / 2 \rho\right)} \mathrm{e}^{\mathrm{i} q_{\mathrm{y}} \mathrm{y}} \tilde{\varepsilon}_{\mathrm{if}}(u, \mathrm{y}) \\
& =\int \mathrm{dz} \mathrm{e}^{\mathrm{i} q_{\mathrm{x}} \mathrm{z}^{2} / 2 \rho} \mathrm{x}(q) \\
& =-(2 \pi \mathrm{i})^{1 / 2}\left(\frac{\rho}{q_{\mathrm{x}}}\right)^{1 / 2} 4 \pi C \cos 2 \varphi_{0} \frac{1}{q^{2}}
\end{aligned}
$$

The essential feature of eq. (D.9) is its proportionality to $1 / q^{2} q_{\mathrm{x}}^{1 / 2}$. For the general $\mathbf{q}$ vectors of interest here $q_{\mathrm{x}} \sim q$ and the intensity $|A|^{2}$ will be proportional to $q^{-5}$.

\section{References}

[1] Sackmann, H., Demus, D., Mol. Crystals 2 (1966) 81.

[2] Friedel, G., Annls. de Phys. 18 (1922) 273.

[3] BRagG, W. L., Suppt to Nature no 3360 (1934) 445.

[4] Bouligand, Y., a) J. Physique 33 (1972) 525 ; b) $J$. Physique 33 (1972) 715.

[5] De Gennes, P. G., C. R. Hebd. Séan. Acad. Sci. B 275 (1972) 549.

[6] Parodi, O., Solid State Commun 11 (1972) 1503.

[7] a) Kasner, E., SuPnick, F., Proc. Nat. Acad. Sc. (USA) 29 (1943) 378.

b) Melzak, Z. A., Canad. J. Math. 18 (1966) 838. HiRST, K. E., J. London Math. Soc. 42 (1967) 281. LARMAN, D. G., J. London Math. Soc. 42 (1967) 292.

c) Eggleton, A., Proc. Cambridge Phil. Soc. 49 (1953) 26.

[8] Gilbert, E. N., Canad. J. Math. 16 (1964) 286.

[9] Frank, F. C., Disc. Faraday Soc. 25 (1958) 19.

[10] De Gennes, P. G., J. Physique 30 (1969) C 465.
[11] Grandjean has observed long ago the texture of a smectic A in contact with a solid layer made of two single crystals with different orientations : this creates a conflict of the type discussed here, and does give rise to a set of focal conics qualitatively similar to figure 8. See GrandJEAN, F. Bull. Soc. Franc. Minéralogie 39 (1916) 164.

[12a] Friedel, J., Kleman, M., J. Physique 30 (1969) C 4 43.

[12b] De Gennes, P. G., C. R. Hebd. Séan. Acad. Sci., B 275 (1972) 939

[13] For a discussion on turbulent eddies, see for instance LANDAU-Lifshitz, Fluid Mechanics (Pergamon Press) 1959.

[14] LandaU, L. D., JETP 13 (1943) 377.

[15] See for instance De GenNes, P. G., Superconductivity of metals and alloys (Benjamin, New York) 1967, Chap. 2.

[16] See for instance De Gennes, P. G., Molecular Crystals 7 (1969) 325. 